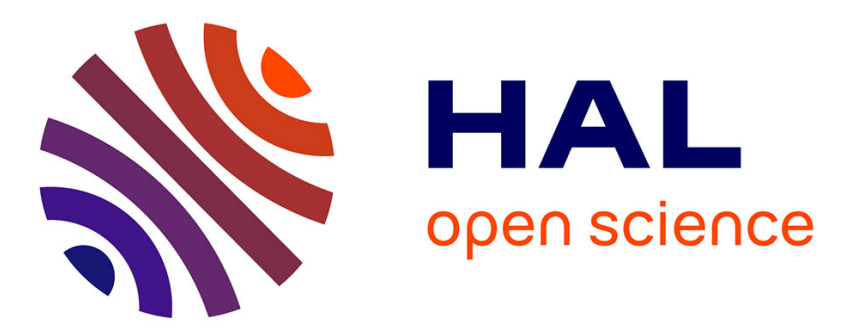

\title{
FSO Communication for High Speed Trains: Blind Data Detection and Channel Estimation
}

Mohammad Taghi Dabiri, Seyed Mohammad Sajad Sadough, Mohammad Ali Khalighi

\section{- To cite this version:}

Mohammad Taghi Dabiri, Seyed Mohammad Sajad Sadough, Mohammad Ali Khalighi. FSO Communication for High Speed Trains: Blind Data Detection and Channel Estimation. International Symposium on Communication Systems, Networks and Digital Signal Processing, Jul 2018, Budapest, Hungary. 10.1109/CSNDSP.2018.8471860 . hal-02423867

\section{HAL Id: hal-02423867 https://hal.science/hal-02423867}

Submitted on 29 Mar 2020

HAL is a multi-disciplinary open access archive for the deposit and dissemination of scientific research documents, whether they are published or not. The documents may come from teaching and research institutions in France or abroad, or from public or private research centers.
L'archive ouverte pluridisciplinaire HAL, est destinée au dépôt et à la diffusion de documents scientifiques de niveau recherche, publiés ou non, émanant des établissements d'enseignement et de recherche français ou étrangers, des laboratoires publics ou privés. 


\title{
FSO Communication for High Speed Trains: Blind Data Detection and Channel Estimation
}

\author{
M. T. Dabiri, S. M. S. Sadough and M. A. Khalighi
}

\author{
Department of Electrical Engineering, Shahid Beheshti University G. C., Tehran, Iran \\ Aix Marseille University, CNRS, Centrale Marseille, Institut Fresnel, Marseille France \\ Email: \{m_dabiri,s_sadough\}@sbu.ac.ir, ali.khalighi@fresnel.fr
}

\begin{abstract}
We consider an free-space optical (FSO) system for high-speed trains where the sampling clock offset is randomly changing and the receiver does not have any information on the instantaneous channel fading coefficient. Based on the use of multiple samplers at the receiver, we propose a novel sequence blind detection method, thus without requiring any pilot symbol. Furthermore, using the detected data, we propose a channel estimation scheme for the considered system.

Index Terms-Free-space optical communications, data detection, time synchronization, atmospheric turbulence.
\end{abstract}

\section{INTRODUCTION}

Free-space optical (FSO) communication is well known as a promising wireless access technology thanks to its feautures of huge available license-free spectrum, low-cost implementation, and robustness to electromagnetic interference [1]. In most commercial FSO systems, intensity modulation with direct detection (IM/DD) is used for the reason of relatively low implementation complexity. When On-Off keying (OOK) modulation is used, the receiver requires adaptive threshold setting for signal detection. In order to avoid data framing and packetization at the transmitter and also the transmission of pilot symbols (leading to a loss of spectral efficiency), several works have considered sequence detection without pilot transmission. In the earlier works, maximum-likelihood sequence detection and its generalization without using pilot symbols were considered in [2], [3]. However, the proposed methods generally suffer from high computational complexity. Therefore, more recent works have tried to design lower complexity signal detection methods, such as [4]-[14].

In this paper we are interested in using the FSO technology for providing high-data-rate wireless access for high speed trains, which has attracted a great deal of attention in the past few years. Indeed, the demand of passengers to access broadband services with high quality in high speed trains is exponentially increasing. However, current radio frequency wireless technologies such as the 4-Generation Long Term Evolution (LTE) (and LTE-Advanced) networks can offer unreliable handovers and limited data rate, resulting in unsatisfying quality-of-experience for users [15]-[17]. Within this context, FSO technology has been recognized as a promising connectivity solution [17]-[19]. However, in most of these works, OOK symbols are detected under the assumption of perfectly synchronized (PSY) transmitter and receiver, which is practically not valid for FSO links with high mobility. In fact, apart from the requirement of highly accurate and agile beam tracking, one of the most important challenges of such system is time synchronization between the transmitter and the receiver. To clarify better this point, consider a high data-rate ground-to-train FSO link with bitrate $R_{b}=1 / T_{b}=1 \mathrm{Gbps}$, where $T_{b}$ is the bit duration, and a train speed of $V_{t}=100 \mathrm{~m} / \mathrm{s}$ (i.e. $360 \mathrm{Km} / \mathrm{h}$ ). Given the speed of light $c=3 \times 10^{8} \mathrm{~m} / \mathrm{s}$, only after $1 \mathrm{~ms}$ the sampling time at the receiver is deviated by at least $0.33 T_{b}$. This can result in a significant performance degradation of the link. Moreover, due to the Doppler effect, the coherence time of the underlying channel is reduced significantly. Hence, for reliable communication we would need to adjust the synchronization at intervals much shorter than $1 \mathrm{~ms}$, which requires the insertion of several pilot bits inside each data frame and the use of extra-fast electrical synchronization circuits. However, this comes at the cost of a considerable spectral efficiency loss and an important increase in the system complexity and cost.

These considerations suggest the practical interest of blind signal detection for such FSO links with high mobility. In this paper, we assume that the sampling clock offset is randomly changing and, in addition, the receiver does not have access to the instantaneous channel fading coefficient. By using $N_{s}$ samplers at the receiver, we propose a sequence blind detection method to detect OOK signals over an observation window of length $L$. The proposed method, which relies on the knowledge of the channel distribution, can achieve a performance close to the receiver with perfect PSY and channel state information (PCSI). In the second part of this paper, we propose an efficient estimation scheme based on the detected data.

The reminder of this paper is organized as follows. In Section II, we describe our system model and main assumptions. Data detection schemes for considered system model is proposed in Section III. Next, in Section IV, we present our numerical results to study the performance of the proposed detection method. Finally, Section V summarizes our work.

\section{SYSTEM MODEL}

We consider an FSO transceiver with high mobility using IM/DD with OOK modulation. As mentioned in the introduction, due to the high mobility and high data rate of the system, the sampling clock offset at the receiver is 


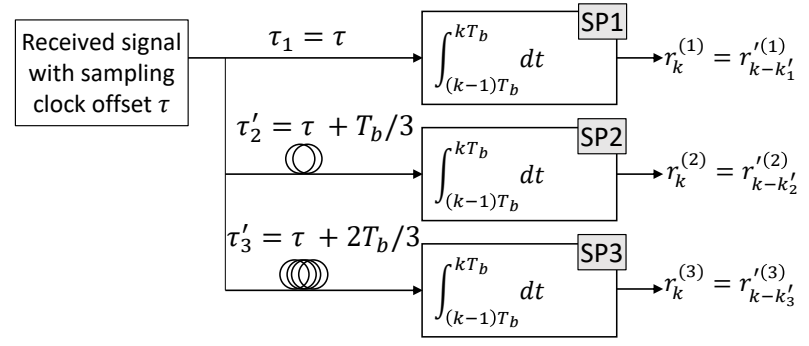

Fig. 1. Block diagram of the receiver samplers for $N_{s}=3$.

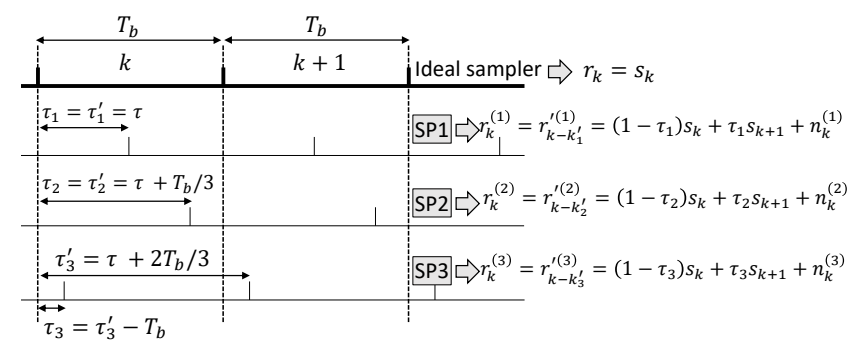

Fig. 2. Receiver samplers corresponding to Fig. 1 assuming that the offset clock between the transmitter and the first sampler (SP1) equals to $\tau$.

considered to be constantly changing, where we use $N_{s}$ samplers denoted by $\mathrm{SP}_{\mathrm{i}}, i \in\left\{1,2, \ldots, N_{s}\right\}$. Let $\tau$ be the sampling clock offset between the transmitter and $\mathrm{SP}_{1}$. We assume that $\tau$ is an unknown parameter and has a uniform distribution in the interval $\left(-T_{b}, T_{b}\right)$. As illustrated in Fig. 1 for $N_{s}=3, \mathrm{SP}_{\mathrm{i}}$ samples the $i$ th integrator output at time $t=\tau_{i}^{\prime}+k T_{b}$ where $\tau_{i}^{\prime}=\tau+(i-1) T_{b} / N_{s}$, with $k$ denoting the $k$ th bit interval. The corresponding output sample can be written as

$$
r_{k}^{\prime(i)}=\eta h\left(1-\tau_{i}\right) s_{k+k_{i}^{\prime}}+\eta h \tau_{i} s_{k+k_{i}^{\prime}+1}+n_{k+k_{i}^{\prime}}^{(i)},
$$

where $\tau_{i}=\tau_{i}^{\prime}-k_{i}^{\prime} T_{b}, k_{i}^{\prime}=\left\lfloor\tau_{i}^{\prime} / T_{b}\right\rfloor$ and $\lfloor$.$\rfloor is the well$ known floor function. Also, $h$ denotes the instantaneous channel coefficient, assumed to remain unchanged over a large number of transmitted bits; $s_{k}$ denotes the transmitted OOK symbols which take the values $2 P_{t}$ or zero for the cases of a bit " 1 " and " 0 ", respectively; $\eta$ denotes the photodetector responsivity; and $n_{k}^{(i)}$ is the zero mean Gaussian noise with variance $N_{0}$, corresponding to thermal and/or background noise [20].

According to (1), the $\mathrm{SP}_{\mathrm{i}}$ 's' output at the discrete time $k$ depends on the transmitted signals at discrete times $k+k_{i}^{\prime}$ and $k+k_{i}^{\prime}+1$. Note that, $k_{i}^{\prime}=\left\lfloor\tau_{i}^{\prime} / T_{b}\right\rfloor$ only takes three discrete values $\{-1,0,1\}$. Moreover, although $k_{i}^{\prime}$ depends on $\tau, k_{i}^{\prime}$ changes much slower than $\tau$. This fact allows us to estimate $k_{i}^{\prime} \mathrm{s}$ with high accuracy compared to the sampling clock offset $\tau$. Here, we reasonably assume that $k_{i}^{\prime}$ is perfectly known at the receiver (the estimation of this parameter is beyond the scope of this paper). Let us denote $r_{k}^{(i)}=r_{k-k_{i}^{\prime}}^{\prime(i)}$. We have

$$
r_{k}^{(i)}=\eta h\left(1-\tau_{i}\right) s_{k}+\eta h \tau_{i} s_{k+1}+n_{k}^{(i)} .
$$

It can be easily seen that one of $\tau_{i} \mathrm{~s}$ gets its value in the interval $\left[0, T_{b} / N_{s}\right)$. This is an important point and, as we will see in the next section, it allows us to simplify the detector's structure. In relation with the illustration of Fig. 1, we have shown the state of the samplers and $r_{k}^{(i)}$ for $N_{s}=3$ in Fig. 2.

\section{Blind Data Detection And Channel ESTIMATION}

We consider a practical scenario where we do not have any prior information on $\tau$ and $h$ and propose a blind detection method, i.e., without requiring any pilot symbol. The proposed method performs block-by-block detection. For this aim, the outputs of $\mathrm{SP}_{\mathrm{i}} \mathrm{s}$ are gathered in the observation windows composed of $L$ intervals. Let $\underline{r}_{i}(L)=$ $\left[r_{1}^{(i)}, r_{2}^{(i)}, \ldots, r_{L}^{(i)}\right]$ be the gathered outputs of $\mathrm{SP}_{\mathrm{i}}$ related to transmitted signals $\underline{s}(L+1)=\left[s_{1}, s_{2}, \ldots, s_{L+1}\right]$. According to (2), the PDF of $\underline{r}_{i}(L)$ conditioned on $h, \tau_{i}$, and $\underline{s}(L+1)$ can be obtained as

$$
p\left[\underline{r}_{i}(L) \mid h, \tau_{i}, \underline{s}(L+1)\right]=\prod_{k=1}^{L} p\left[r_{k}^{(i)} \mid h, \tau_{i}, s_{k}, s_{k+1}\right],
$$

where

$$
\begin{aligned}
p\left[r_{k}^{(i)} \mid h, \tau_{i}, s_{k}, s_{k+1}\right]=\frac{1}{\sqrt{2 \pi N_{0}}} & \\
& \times \exp \left(-\frac{\left|r_{k}^{(i)}-\eta h\left(1-\tau_{i}\right) s_{k}-\eta h \tau_{i} s_{k+1}\right|^{2}}{2 N_{0}}\right) .
\end{aligned}
$$

As explained previously, one of $\tau_{i} \mathrm{~s}, i \in\left\{1, \ldots, N_{s}\right\}$, gets its value in the interval $\left[0, T_{b} / N_{s}\right)$; yet, we don not know which one (which $i$ ). Therefore, we propose here a detection method to decide jointly on $i$ and $\underline{s}(L)$ based on the generalized likelihood ratio test (GLRT) principle as [21], [22]

$$
\begin{aligned}
\hat{i}, \hat{s}(L)= & \underset{i, \underline{s}(L+1)}{\arg \max } p\left[\underline{r}_{i}(L) \mid \tau_{i} \in\left[0, \frac{T_{b}}{N_{s}}\right), \underline{s}(L+1)\right] \\
= & \underset{i, \underline{s}(L+1)}{\arg \max } \int_{0}^{\infty} p\left[\underline{r}_{i}(L) \mid h, \tau_{i} \in\left[0, \frac{T_{b}}{N_{s}}\right), \underline{s}(L+1)\right] \\
& \times f_{h}(h) d h
\end{aligned}
$$

where

$$
\begin{aligned}
p\left[\underline{r}_{i}(L) \mid h, \tau_{i} \in\left[0, \frac{T_{b}}{N_{s}}\right), \underline{s}(L+1)\right] \\
=\frac{N_{s}}{T_{b}} \int_{0}^{\frac{N_{s}}{T_{b}}} p\left[\underline{r}_{i}(L) \mid h, \tau_{i}, \underline{s}(L+1)\right] d \tau_{i} \\
=\frac{N_{s}}{T_{b}} \int_{0}^{\frac{N_{s}}{T_{b}}} \prod_{k=1}^{L} p\left[r_{k}^{(i)} \mid h, \tau_{i}, s_{k}, s_{k+1}\right] d \tau_{i} .
\end{aligned}
$$




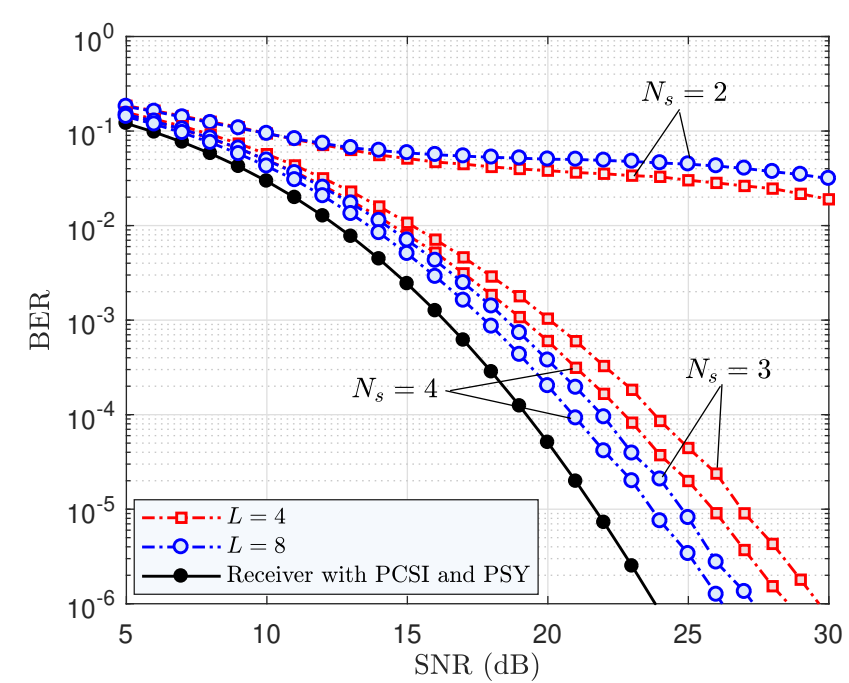

Fig. 3. BER of proposed blind detector versus SNR for different values of $L$ and $N_{s}$.

Substituting (4) in (6) and after some analytical calculations, the closed form expression of (6) is derived as

$$
\begin{aligned}
p\left[\underline{r}_{i}(L) \mid h, \tau_{i} \in\left[0, \frac{T_{b}}{N_{s}}\right), \underline{s}(L+1)\right] \\
=\frac{\mathcal{D}_{1}}{\sqrt{\mathcal{D}_{3}}} Q\left(\frac{N_{s} \sqrt{\mathcal{D}_{3}}}{T_{b} \sqrt{N_{0}}}-\frac{\mathcal{D}_{2}}{\mathcal{D}_{3}}\right),
\end{aligned}
$$

where

$$
\begin{gathered}
\mathcal{D}_{1}=\frac{N_{s} \exp \left(\frac{\mathcal{D}_{2}^{2} / \mathcal{D}_{3}-\sum_{k=1}^{L}\left(r_{k}^{(i)}-\eta h s_{k}\right)^{2}}{2 N_{0}}\right)}{T_{b}\left(2 \pi N_{0}\right)^{L / 2-1}} \\
\mathcal{D}_{2}=\eta h \sum_{k=1}^{L}\left(r_{k}^{(i)}-\eta h s_{k}\right) s_{d, k} \\
\mathcal{D}_{3}=\eta^{2} h^{2} \sum_{k=1}^{L} s_{d, k}^{2}, \quad s_{d, k}=s_{k+1}-s_{k} .
\end{gathered}
$$

We note that the proposed method detects $\underline{\hat{s}}(L)$ by searching over $N_{s} \times 2^{L+1}$ possible states of (5). An important point is that this method relies on the knowledge of $f_{h}(h)$. This would be a secondary issue as the channel distribution, or in other words, the turbulence characteristics, can be determined or known a priori. Nevertheless, for the sake of completeness, we propose here a method to determine the fading statistics.

We assume that the fading statistics are modeled by log-normal distribution with parameter $\sigma_{\mathrm{LN} h}^{2}$, which is a valid assumption for relatively short link spans and/or for a receiver with a relatively large aperture [23], [24]. In the case of changing turbulence conditions, $\sigma_{\mathrm{LN} h}^{2}$ changes with a coherence time much larger than the coherence time of the instantaneous channel coefficient $h$. Let us consider $N_{h}$ consecutive blocks of transmitted symbols and denote by $\hat{h}_{m}, m \in\left\{1, \ldots, N_{h}\right\}$, the estimated $h$ on the $m$ th block. We can estimate $\sigma_{\mathrm{LN} h}^{2}$ using $N_{h}$ estimations of $\hat{h}_{m}$ as

$$
\hat{\sigma}_{\mathrm{LN} h}^{2}=\frac{1}{N_{h}} \sum_{m=1}^{N_{h}}\left(\ln \hat{h}_{m}-\frac{1}{N_{h}} \sum_{m=1}^{N_{h}} \ln \hat{h}_{m}\right)^{2} .
$$

Now, to obtain $\hat{h}_{m}$, we propose an efficient channel estimation method based on the detected data $\underline{\hat{s}}(L)$. For this, given $\underline{s}(L)$ and $\tau_{i}$, we estimate the channel over each block based on the maximum likelihood (ML) criterion as

$$
\begin{aligned}
\hat{h} & =\underset{h}{\arg \max } p\left(\underline{r}_{i}(L-1) \mid h, \tau_{i}, \underline{s}(L)\right) \\
& =\underset{h}{\arg \min } \sum_{k=1}^{L}\left|r_{k}^{(i)}-\eta h\left(1-\tau_{i}\right) s_{k}-\eta h \tau_{i} s_{k+1}\right|^{2} .
\end{aligned}
$$

To obtain the optimum $h$ from (9), we differentiate it with respect to $h$ and set the result to zero. We have

$$
\hat{h}=\frac{\sum_{k=1}^{L-1} r_{k}^{(i)}\left(\left(1-\bar{\tau}_{i}\right) \hat{s}_{k}+\bar{\tau}_{i} \hat{s}_{k+1}\right)}{\eta \sum_{k=1}^{L-1}\left(\left(1-\bar{\tau}_{i}\right) \hat{s}_{k}+\bar{\tau}_{i} \hat{s}_{k+1}\right)^{2}} .
$$

From the previous section, the mean and the variance of $\tau_{i}$ are $\bar{\tau}_{i}=T_{b} / 2 N_{s}$ and $\sigma_{\tau_{i}}^{2}=T_{b}^{2} / 3 N_{s}^{2}$. Obviously, $\sigma_{\tau_{i}}^{2}$ is a decreasing function of $N_{s}$. As a result, $h$ can be estimated from (10) in off-line by substituting $\underline{\hat{s}}(L)$ and $\bar{\tau}$ by $\underline{s}(L)$ and $\tau_{i}$, respectively.

\section{Simulation Results}

In this section, we study the performance of the proposed blind detection method. We assume that the channel is lognormal distributed with $\sigma_{\mathrm{LN} h}^{2}=0.1$, and, to normalize the channel coefficients, we set $\mu_{\mathrm{LN} h}=-\sigma_{\mathrm{LN} h}^{2}$. We also normalize the average transmit optical power, i.e., $\bar{P}_{t}=1$, and consider the electrical signal-to-noise ratio (SNR) as $\frac{2}{N_{0}}$.

In Fig. 3, we have presented bit-error-rate (BER) plots as a function of SNR, to demonstrate the impact of the main parameters, i.e., the number of samplers $N_{s}$, and the length of the observation window, $L$. The BER plot with PCSI and PSY is also provided as the lower bound benchmark. We notice that, as expected, by increasing $L$ and $N_{s}$, the performance of the blind detector is improved. This improvement is significant when $N_{s}$ increases from 2 to 3 . For $N_{s}>2$, the receiver with a larger $L$ has a better performance, but this is the inverse for $N_{s}=2$, where an increase of $L$ results in a performance degradation. From Fig. 3, $L=8$ and $N_{s}=4$ can be considered as suitable choices for the proposed detector as it can achieve a performance close to the receiver with PCSI and PSY, i.e., with an SNR gap of less than $2 \mathrm{~dB}$ at a target BER of $10^{-6}$.

To investigate the efficiency of the proposed channel estimator in (10), we have further shown in Fig. 4, the normalized mean-squared estimation error (MSE) for different values of $N_{s}$ and for $L=4,8$. We have also provided as benchmark the MSE plot for the case of the ML estimator for which $\underline{s}_{i}$ and $\tau_{i}$ are perfectly known. We notice from these results that, as expected, by increasing $L$ and $N_{s}$, the performance of the proposed estimator gets improved and becomes closer to the 'ideal' estimator. 


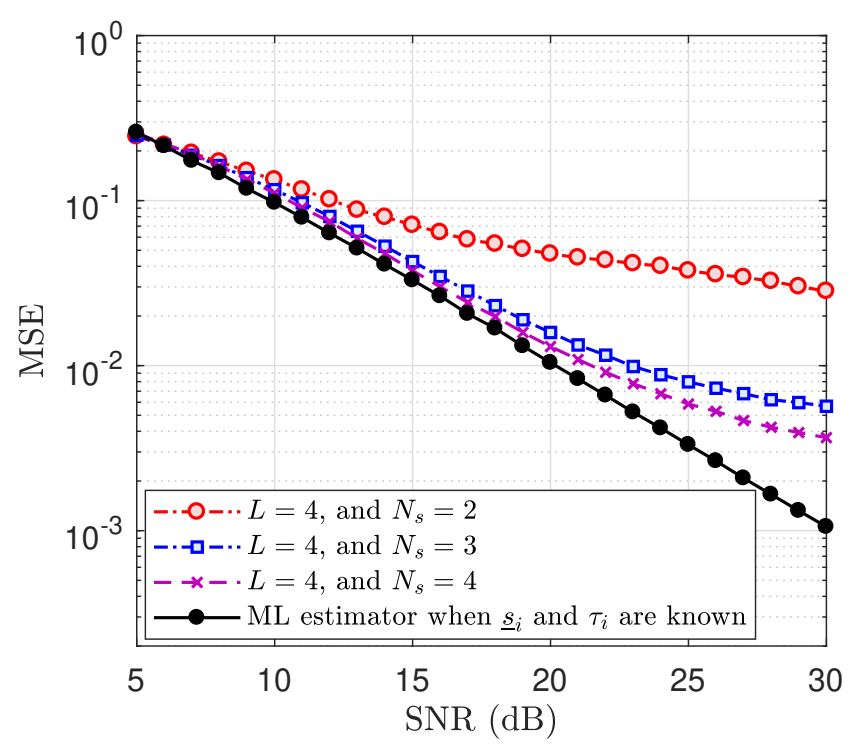

(a) $L=4$

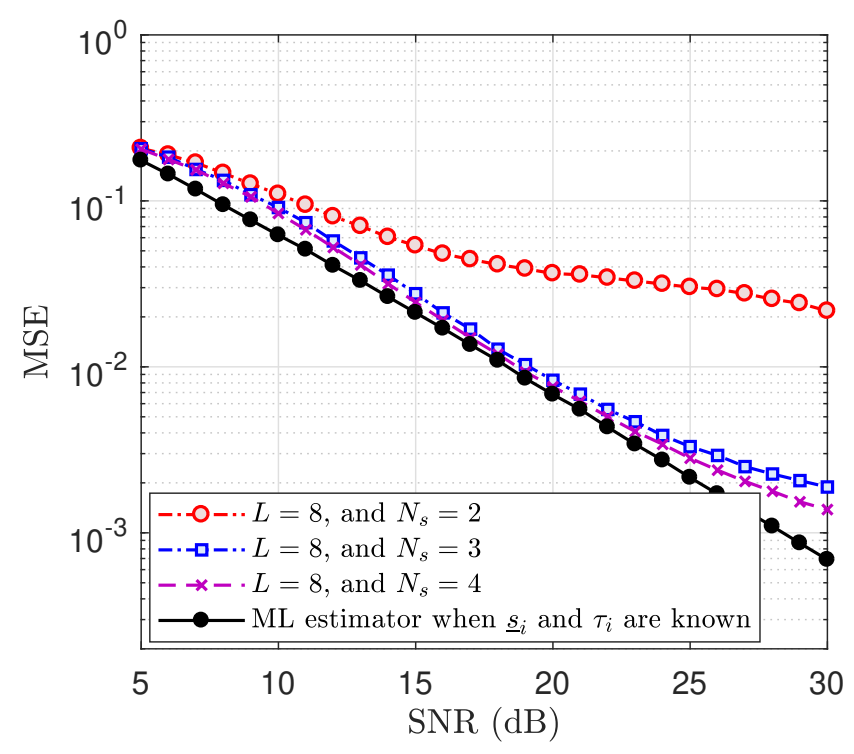

(b) $L=8$

Fig. 4. MSE of the proposed estimator based on (10) for different $N_{s}$; comparison with the ML estimator with known $\underline{s}_{i}$ and $\tau_{i}$.

\section{CONCLUding REMARKS}

In this work, we considered an FSO link with high mobility and investigated optimal signal detection in the case of random sampling clock offset and when the receiver does not have any information on the instantaneous channel fading coefficient. Using $N_{s}$ samplers at the receiver, we proposed a GLRT-based sequence blind detector for OOK signal detection, hence requiring no pilot symbol. Moreover, using the detected data, we proposed an efficient channel estimation scheme for the considered system. By adjusting appropriately the parameters of the receiver, namely the number of samplers $N_{s}$ and the block length $L$, we showed that the proposed detector can achieve performance close to the receiver with PSY and PCSI.
Despite showing a good performance, the computational complexity of the proposed method remains relatively high. In fact, in order to detect a sequence of $L$ symbols, the proposed method requires to calculate $N_{s} \times 2^{L+1}$ possible states of the GLRT-based metric $\left(3 \times 2^{8+1}=1536\right.$ for $N_{s}=3$ and $L=8$, considered here). Note that each metric calculation requires solving an integral over channel distribution, whose accuracy highly depends on the estimation of the channel distribution $f_{h}(h)$ at the receiver. Therefore, although being quite promising, the proposed methods should be simplified in order to be adapted for implementation in a practical highrate FSO system, which is the subject of our future research.

\section{REFERENCES}

[1] M. A. Khalighi and M. Uysal, "Survey on free space optical communication: A communication theory perspective," IEEE Communications Surveys Tutorials, vol. 16, no. 4, pp. 2231-2258, 2014.

[2] X. Zhu and J. M. Kahn, "Free-space optical communication through atmospheric turbulence channels," IEEE Transactions on Communications, vol. 50, no. 8, pp. 1293-1300, 2002.

[3] M. L. Riediger, R. Schober, and L. Lampe, "Fast multiple-symbol detection for free-space optical communications," IEEE Transactions on Communications, vol. 57, no. 4, pp. 1119-1128, 2009.

[4] T. Song and P.-Y. Kam, "A robust GLRT receiver with implicit channel estimation and automatic threshold adjustment for the free space optical channel with IM/DD," Journal of Lightwave Technology, vol. 32, no. 3, pp. 369-383, 2014.

[5] — " "Robust data detection for the photon-counting free-space optical system with implicit CSI acquisition and background radiation compensation," Journal of Lightwave Technology, vol. 34, no. 4, pp. 1120-1132, 2016.

[6] M. M. Abadi, Z. Ghassemlooy, M.-A. Khalighi, S. Zvanovec, and M. R. Bhatnagar, "FSO detection using differential signaling in outdoor correlated-channels condition," IEEE Photonics Technology Letters, vol. 28, no. 1, pp. 55-58, 2016.

[7] M. M. Abadi, Z. Ghassemlooy, S. Zvanovec, M. R. Bhatnagar, M.A. Khalighi, and Y. Wu, "Impact of link parameters and channel correlation on the performance of FSO systems with the differential signaling technique," Journal of Optical Communications and Networking, vol. 9, no. 2, pp. 138-148, 2017.

[8] X. Liu, C. Gong, and Z. Xu, "Sequential detection for optical wireless scattering communication," Journal of Optical Communications and Networking, vol. 7, no. 9, pp. 86-95, 2017.

[9] L. Yang, B. Zhu, J. Cheng, and J. F. Holzman, "Free-space optical communications using on-off keying and source information transformation," Journal of Lightwave Technology, vol. 34, no. 11, pp. 2601-2609, 2016.

[10] S. Ding, J. Zhang, and A. Dang, "Adaptive threshold decision for onoff keying transmission systems in atmospheric turbulence," Optics express, vol. 25, no. 20, pp. 24425-24436, 2017.

[11] M. T. Dabiri, S. M. S. Sadough, and M. A. Khalighi, "FSO channel estimation for OOK modulation with APD receiver over atmospheric turbulence and pointing errors," Optics Communications, vol. 402, pp. 577-584, 2017.

[12] M. T. Dabiri and S. M. S. Sadough, "Performance analysis of EMbased blind detection for ON-OFF keying modulation over atmospheric optical channels," Optics Communications, 2018.

[13] M. T. Dabiri, S. M. S. Sadough, and H. Safi, "GLRT-based sequence detection of OOK modulation over FSO turbulence channels," IEEE Photon. Technol. Lett., vol. PP, no. 99, pp. 1-1, 2017.

[14] M. T. Dabiri and S. M. S. Sadough, "Generalized blind detection of OOK modulation for free-space optical communication," IEEE Communications Letters, vol. PP, no. 99, pp. 1-1, 2017.

[15] B. Ai et al., "Challenges toward wireless communications for highspeed railway," IEEE Transactions on Intelligent Transportation Systems, vol. 15, no. 5, pp. 2143-2158, 2014.

[16] A. Sniady and J. Soler, "LTE for railways: Impact on performance of ETCS railway signaling," IEEE Vehicular Technology Magazine, vol. 9, no. 2, pp. 69-77, 2014. 
[17] S. Fathi-Kazerooni, Y. Kaymak, R. Rojas-Cessa, J. Feng, N. Ansari, M. Zhou, and T. Zhang, "Optimal positioning of ground base stations in free-space optical communications for high-speed trains," IEEE Transactions on Intelligent Transportation Systems, 2017.

[18] Q. Fan, M. Taheri, N. Ansari, J. Feng, R. Rojas-Cessa, M. Zhou and T. Zhang, "Reducing the impact of handovers in ground-to-train free space optical communications," IEEE Transactions on Vehicular Technology, 2017.

[19] R. Paudel, X. Tang, and Z. Ghassemlooy, "Laboratory demonstration of FSO ground-to-train communications with multiple base stations,' in Communication Systems, Networks and Digital Signal Processing (CSNDSP), International Symposium on. IEEE, 2016, pp. 1-6.

[20] F. Xu, M. A. Khalighi, and S. Bourennane, "Impact of different noise sources on the performance of PIN- and APD-based FSO receivers," COST IC0802 Workshop, IEEE ConTEL Conference, pp. 211-218,
June 2011, Graz, Austria.

[21] D. Warrier and U. Madhow, "Spectrally efficient noncoherent communication," IEEE Transactions on Information Theory, vol. 48, no. 3, pp. 651-668, 2002.

[22] M. Wu and P. Y. Kam, "Performance analysis and computational complexity comparison of sequence detection receivers with no explicit channel estimation," IEEE Transactions on Vehicular Technology, vol. 59, no. 5, pp. 2625-2631, 2010.

[23] M. A. Khalighi, N. Schwartz, N. Aitamer, and S. Bourennane, "Fading reduction by aperture averaging and spatial diversity in optical wireless systems," IEEE/OSA Journal of Optical Communications and Networking, vol. 1, no. 6, pp. 580-593, Nov. 2009.

[24] A. Mostafa and S. Hranilovic, "Channel measurement and markov modeling of an urban free-space optical link," Journal of Optical Communications and Networking, vol. 4, no. 10, pp. 836-846, 2012. 